\title{
C30F12.4 influences oogenesis, fat metabolism, and lifespan in C. elegans
}

\author{
Lu Wang ${ }^{1}$, Fei $X^{2}$, Guishuan Wang ${ }^{1}$, Xiaorong Wang ${ }^{1}$, Ajuan Liang ${ }^{1}$, Hefeng Huang ${ }^{1 凶}$, Fei Sun $^{1 凶}$ \\ ${ }^{1}$ International Peace Maternity \& Child Health Hospital, Shanghai Key laboratory for Reproductive Medicine, School of \\ Medicine, Institute of Embryo-Fetal Original Adult Disease, Shanghai Jiaotong University, Shanghai 200030, China \\ 2 School of Life Sciences, University of Science and Technology of China, Hefei 230026, China \\ $\triangle$ Correspondence: huanghefg@sjtu.edu.cn (H. Huang), sunfei@shsmu.edu.cn (F. Sun) \\ Received April 20, 2016 Accepted July 19, 2016
}

\begin{abstract}
Reproduction, fat metabolism, and longevity are intertwined regulatory axes; recent studies in $C$. elegans have provided evidence that these processes are directly coupled. However, the mechanisms by which they are coupled and the reproductive signals modulating fat metabolism and lifespan are poorly understood. Here, we find that an oogenesis-enriched gene, c30f12.4, is specifically expressed and located in germ cells and early embryos; when the gene is knocked out, oogenesis is disrupted and brood size is decreased. In addition to the reproductive phenotype, we find that the loss of c30f12.4 alters fat metabolism, resulting in decreased fat storage and smaller lipid droplets. Meanwhile, c30f12.4 mutant worms display a shortened lifespan. Our results highlight an important role for c30f12.4 in regulating reproduction, fat homeostasis, and aging in $C$. elegans, which helps us to better understand the relationship between these processes.
\end{abstract}

KEYWORDS C30F12.4, oogenesis, fat metabolism, lifespan

\section{INTRODUCTION}

Reproduction, fat metabolism, and longevity are interconnected with each other, and numerous observations have suggested that reproduction can influence organismal lifespan and aging (Hansen et al., 2013). In many species, an abolished or reduced reproductive system can increase fat

Electronic supplementary material The online version of this article (doi:10.1007/s13238-016-0308-z) contains supplementary material, which is available to authorized users. storage and lead to weight gain (Corona et al., 2009; Judd et al., 2011). In C. elegans, signals from the reproductive system can regulate longevity, and ablation of germ cells can alter fat metabolism and significantly prolong lifespan (Hsin and Kenyon, 1999; Goudeau et al., 2011). Recent studies have provided the most direct molecular evidence in C. elegans for the connection between reproduction, fat metabolism, and lifespan, showing that germline removal not only affects fat metabolism but also extends lifespan profoundly (Goudeau et al., 2011; McCormick et al., 2012; Wollam et al., 2012; Khanna et al., 2014).

Several pathways have been indicated to have important roles in coupling reproduction, fat metabolism, and life span. One of these is the insulin/IGF-1 signaling pathway: in $C$. elegans, one of the most important components of the insulin/IGF-1 signaling pathway is daf-16, a FOXO transcription factor that has an essential role in lifespan extension upon germline loss (Lin et al., 1997; Berman and Kenyon, 2006; Kenyon, 2010). Another is the steroid/NHR signaling pathway: this pathway includes many regulators, such as daf-12, a nuclear hormone receptor, and daf-9, a cytochrome P450 similar to CYP27A1. These two regulators are specific to germline signaling in their longevity-promoting effects because daf-9 mutant worms display an extended lifespan, and loss-of-function mutations in daf-12 increase lifespan in males (Gems et al., 1998; Jia et al., 2002; McCormick et al., 2012). Other reports also suggest that insulin/IGF-1 and steroid hormone/NHR signaling interact with each other to promote lifespan extension in germlineablated worms (Berman and Kenyon, 2006).

Even though the existence of multiple links between reproduction, fat metabolism, and lifespan has been supported in so many works, these are still many intriguing questions to be addressed. In this study, we found that the protein, C30F12.4, from reproductive system and early embryo, could regulate fat 
homeostasis and lifespan in C. elegans. Our results define the physiological roles for C30F12.4 in regulating oogenesis, fat storage, the size of lipid droplets, and aging.

\section{RESULTS}

\section{Using dual sgRNAs to knock out c30f12.4}

C30F12.4, which has been reported to be a strictly maternal gene, is one of the oogenesis-enriched genes of heretofore unknown function (Spencer et al., 2011). RNAi inactivation of c30f12.4 resulted in significantly decreased brood size in hermaphrodites (Fig. S1), prompting us to study the function of c30f12.4 in regulating worm development. To further characterize c30f12.4, we sought to generate null alleles using CRISPR/Cas9 technology with dual sgRNAs.

We coinjected sgRNAs targeting exons 2 and 3 of c30f12.4, Cas9 and mCherry expression plasmids into young adult N2 worms (Fig. 1A). F1 animals expressing mCherry were first transferred to NGM plates. After three days, F1 with corresponding F2 progeny were harvested and screened by PCR amplification. We identified lesions in the c30f12.4 gene consistent with Cas9-directed cleavage (Fig. 1B). This deletion was further confirmed by PCR amplification of DNA, mRNA expression, and DNA sequencing (Fig. 1B-D). The large deletion may reflect $a$ simultaneous cleavage directed by the two sgRNAs, whose targets are separated by 378 bases in this experiment. Nevertheless, we found no difference in movement, appearance, and growing rate in the first three days post hatch between $\mathrm{N} 2$ and c30f12.4 mutant animals (Fig. 1E).

\section{Disruption of c $30 f 12.4$ causes female sterility in worms}

As mentioned above, treating animals with RNAi to reduce c30f12.4 levels resulted in decreased brood size in worms (Fig. S1). We therefore first examined the progeny of the c30f12.4 (ust043), which exhibited a 3-fold decrease in brood size at $20^{\circ} \mathrm{C}$ in hermaphrodites (Fig. 2A). Moreover, when we crossed the male and female c30f12.4 (ust043) and fog-2 (JK574) worms, we found that the progeny of the

A

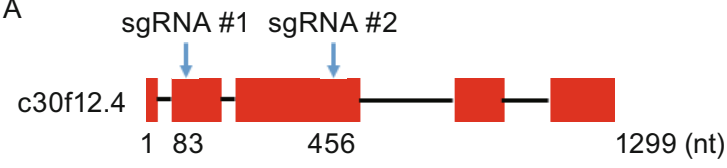

B

$84 \quad$ sgRNA \#1 PAM

sgRNA \#2 PAM

479

Wild type GAGCCCCCACTCACGCCTGATGG (350 bp) GATGACTCAGATGGCTCAAATGG Mutant GAGCCCCCACTCAC -AATGG (-378 bp)

C

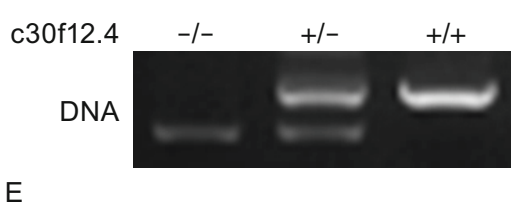

E

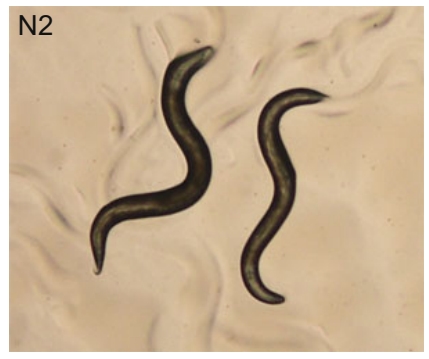

D

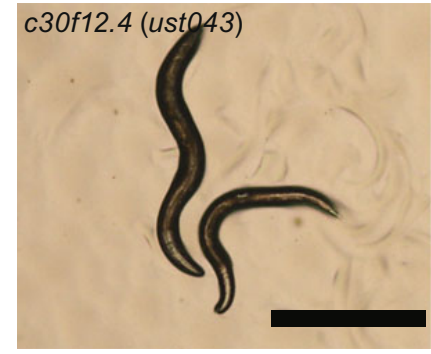

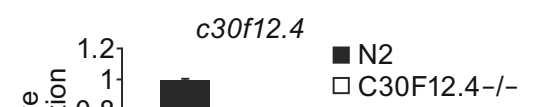

C $30 \mathrm{~F} 12.4-1-$

Figure 1. Dual sgRNA-guided deletion of the c30f12.4 gene. (A) Schematic of the $c 30 f 12.4$ gene. Positions of sgRNA-guided cleavage sites are indicated. (B) Sequence alignments of the c30f12.4 gene in wild-type and mutant worms. The sgRNA PAM sequence is labeled in red. The number of deleted (-) bases is shown to the right of each indel. The numbers in parentheses within the sequence represent the number of bases not shown. Numbers on the top of sequences indicate positions relative to the transcription start site. (C) PCR amplification of the targeted region in N2 and c30f12.4 deletion mutant. (D) PCR amplification and real time PCR analysis of $c 30 f 12.4$ gene expression in total RNAs from the N2 and $c 30 f 12.4$ mutant. ${ }^{* * *} P<0.001, t$-test. (E) The phenotype between $\mathrm{N} 2$ and c30f12.4 mutant. Scale bar $=0.5 \mathrm{~mm}$. 

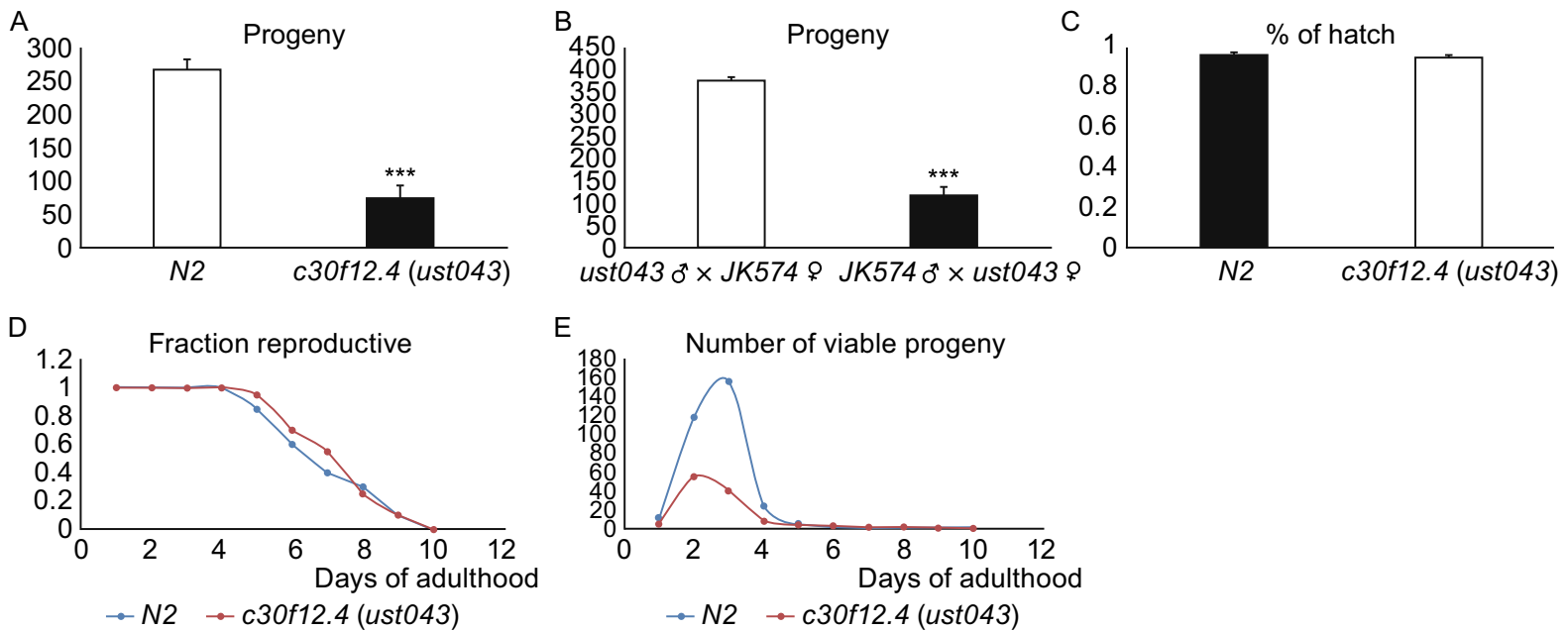

Figure 2. Disruption of $c 30 f 12.4$ causes female sterility in worms. (A) Brood size of wild-type or mutant worms was determined at $20{ }^{\circ} \mathrm{C}$. The x-axis indicates corresponding genotypes; the y axis is the mean value of total number of progeny per animal. Greater than six animals per trial, average of three trials. ${ }^{* * *} P<0.001, t$-test. (B) The number of progeny after mating between fog-2 (JK574) and $c 30 f 12.4$ (ust043). Four animals per trial, average of three trials. ${ }^{* * *} P<0.001, t$-test. (C) Percentage of embryos hatched after laying from day two wild-type and c30f12.4 adults. Greater than 500 eggs per trial, average of three trials. (D) Reproductive span of wild-type and c30f12.4. Ten animals per trial, average of three trials. (E) C30f12.4 mutants produce fewer early and total progeny than wild type. Ten animals per trial, average of three trials.

JK574 male $\times$ ust043 female cross exhibited a 3-fold decrease relative to the $J K 574$ female $\times$ ust043 male cross (Fig. 2B), indicating that knockout of c30f12.4 may only influence oogenesis, but not spermatogenesis. In order to confirm that a dysfunction in oogenesis rather than hatch led to decreased brood size, we examined the hatch rate in mutant and wild-type worms. As expected, the hatch rates of c30f12.4 mutants were comparable to those of the wild type (Fig. 2C). Meanwhile, the onset of progeny production in c30f12.4 mutants was not delayed and progeny was steadily produced at the age when reproduction ceased in the wild type (Fig. 2D and 2E). The overall difference was that c30f12.4 mutants produced fewer early and total progeny over the same period of time (Fig. 2E). During meiosis, a major spatial reorganization of chromosomes within nuclei occurs in the transition zone region of the germ line, corresponding to the leptotene/zygotene stages of meiotic prophase (Dernburg et al., 1998). The chromatin becomes asymmetrically localized and concentrated toward one side of the nucleus, generating polarity that imparts a distinctive crescent-shaped appearance to the Hoechst-stained chromatin in transition zone nuclei that is readily evident, even in low magnification images (Fig. 3A). Nonetheless, in the c30f12.4 germ line, we could not find any crescent-shaped nuclei in any region of the gonad (Fig. 3B).

\section{Loss of c30f12.4 alters lipid metabolism}

Besides the function of c30f12.4 in influencing oogenesis in worms, we also found that the gene could affect fat storage. We compared the fat content between the wildtype and c30f12.4 mutant worms by fixed Nile Red staining when they were fed with the standard Escherichia coli OP50 diet ad libitum. Mutation of c30f12.4 led to a significant, $23 \%$ reduction of intracellular lipids compared with wild-type worms (Fig. 4A). Meanwhile, loss of c30f12.4 resulted in a smaller size of lipid droplets (Fig. 4B). In order to further confirm the role of c30f12.4 in regulating fat homeostasis in living worms, we crossed c30f12.4 mutant worms with hjSi56, which can express GFP-DGAT2, a marker of lipid droplets (Xu et al., 2012; Klemm et al., 2013). In wild-type worms, the lipid droplets are clear, bright, and relatively large, while in mutant worms, they are blurry and smaller (Fig. 5A and 5B). Moreover, we found that the expression of fat -5 and fat -7 were decreased in mutant worms (Fig. S2). These results indeed demonstrated that c30f12.4 could affect fat homeostasis. Next, we also asked whether mutation of c30f12.4 could influence life span because of its role in regulating fat metabolism. We found that c30f12.4 mutant worms displayed significantly reduced animal survival (Fig. 6A). Moreover, we found that vacuole-like structures appeared in mutant worm bodies towards the end of the reproductive period from the seventh day of adulthood, which indicates an aging worm (Herndon et al., 2002), while in normal worms, there are no such structures (Fig. 6B). The phenotype is associated with the decreased life span we detected in mutant worms. These results indicated that the disrupted fat homeostasis in c30f12.4 mutant worms may lead to aging and a shortened life span. 


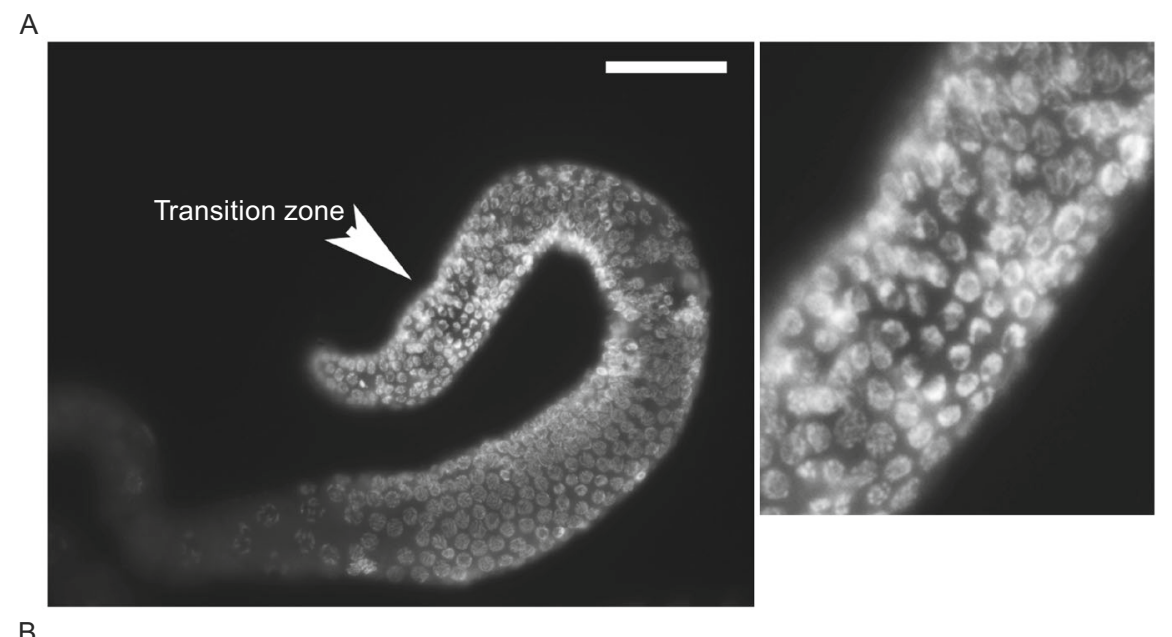

B

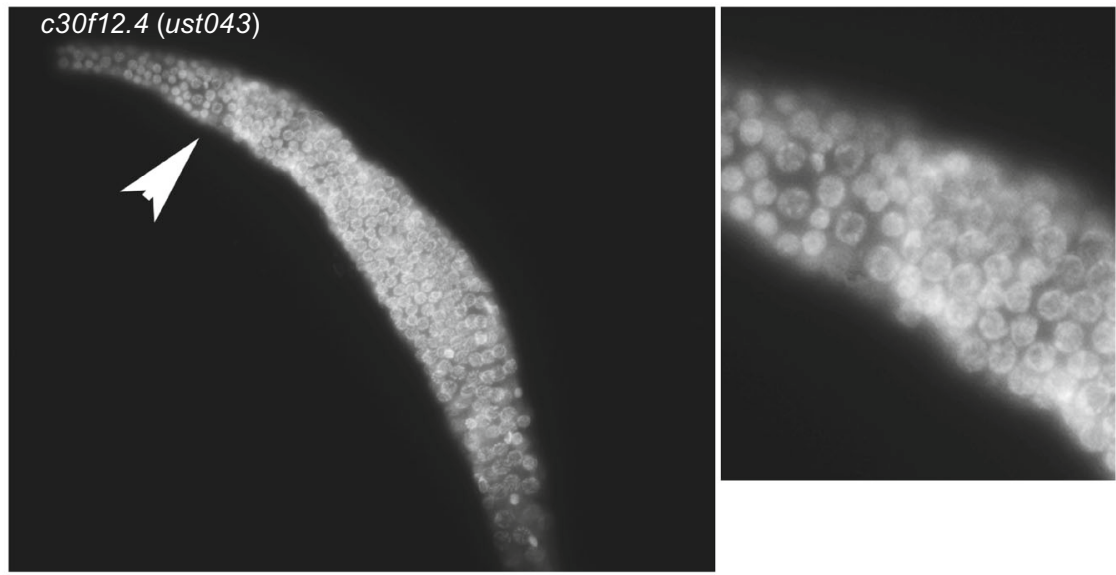

Figure 3. Absence of transition zone in c30f12.4 mutant worms. Each panel shows Hoechst-stained nuclei in an isolated gonad. (A) Wild-type gonad, arrowhead: transition zone. (B) C30f12.4 mutant gonad. Scale bar $=50 \mu \mathrm{m}$.

\section{C30F12.4 are specifically expressed in germ cells and early embryos}

To detect the endogenously produced protein encoded by c30f12.4 and the location of C30F12.4 in worms, we constructed a 3×FLAG-GFP:C30F12.4 transgene worm using the Mos1-mediated single-copy insertion (mossCl) system. We found that the fused GFP::C30F12.4 was expressed very well and could be detected clearly under the green fluorescent protein channel. C30F12.4 was expressed and located specifically in germ cells and early embryos, especially in oocytes and single-cell embryos in worms (Fig. S3). Moreover, this transgene can rescue the decreased brood size of c30f12.4 mutant worms from 83 to 227 (Fig. S4). In order to further identify the localization of C30F12.4 in germ cells and embryos, we isolated gonads and embryos and found that C30F12.4 was mainly located in the cytoplasm around the germ cells and everywhere in oocytes (Fig. 7C). Meanwhile, we found that C30F12.4 displayed a punctate signal in single-cell embryos, and the expression of C30F12.4 was decreased gradually during embryo development (Fig. 7D).

\section{DISCUSSION}

In this paper, we have identified C. elegans C30F12.4 as the special regulator that links reproduction, fat metabolism, and lifespan. C30F12.4 was reported as an oogenesis-enriched gene, and we found that it was indeed expressed and located in germ cells and early embryos. When this gene was knocked out, oogenesis was disrupted with a missed transition zone, leading to decreased brood size. Meanwhile, fat metabolism in the mutant worms was also influenced: in mutant worms, fat storage was decreased and lipid droplets became smaller, concomitant with a shortened lifespan.

As reported in many papers, reproduction, fat metabolism, and lifespan are interconnected. Germline signals can modulate the activity of an insulin/IGF-1 (insulin-like growth factor) pathway, which has an important role in regulating aging. If the cells that give rise to the germ line are killed or germline stem cells (GSC) undergo cell cycle arrest, the lifespan of the worms is extended (Hsin and Kenyon, 1999; Wang et al., 2008). However, no paper has reported that a protein specifically expressed in the germline can influence not only oogenesis but also fat metabolism and lifespan. 

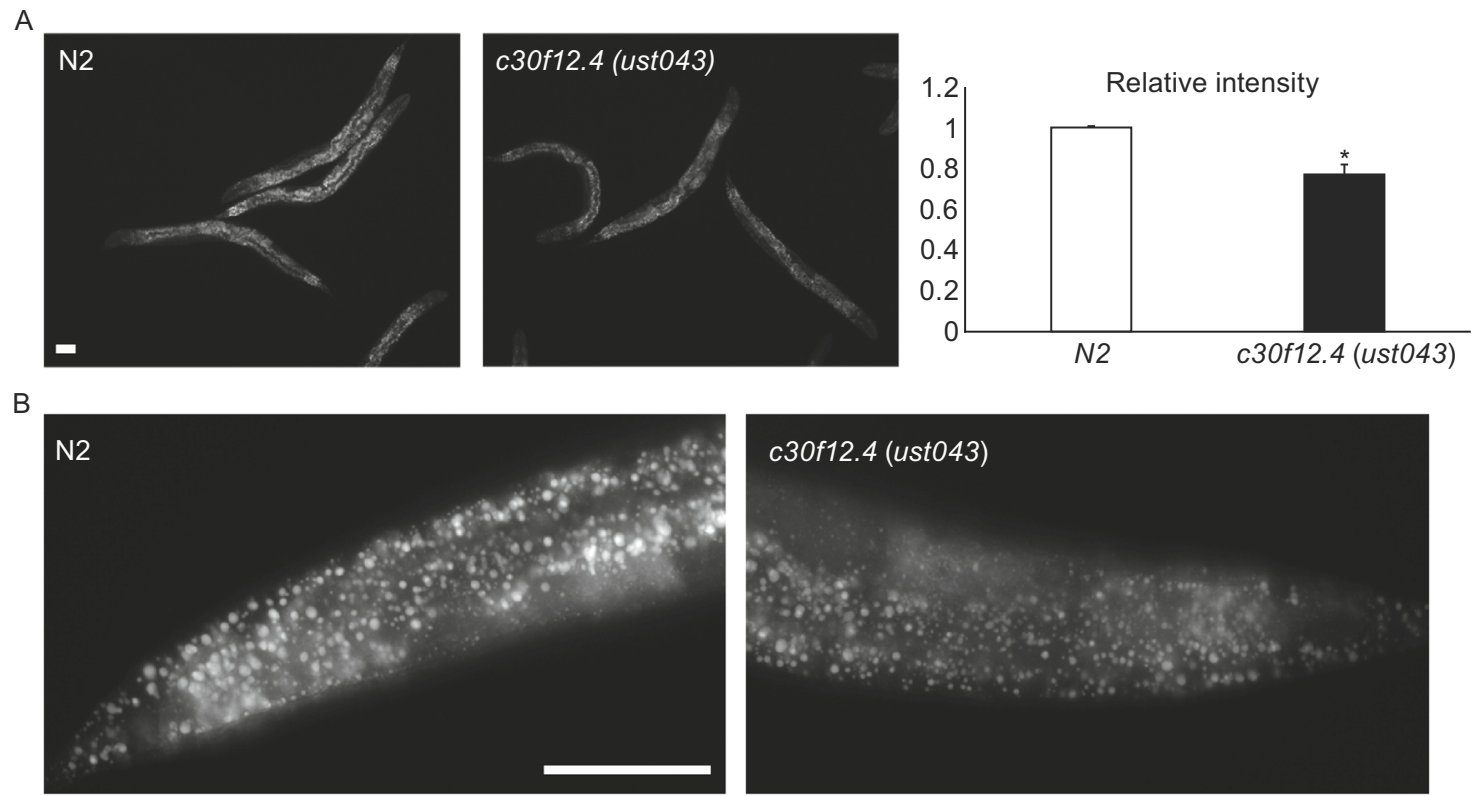

Figure 4. Loss of c $30 f 12.4$ altered fat storage in C. elegans. Late L4 stage worms were fixed with $40 \%$ isopropanol and stained with Nile Red. Images were captured using identical settings and exposure time for each image. Loss of $c 30 f 12.4$ lead to decreased fat storage (A) and smaller lipid droplets (B). ${ }^{*} P<0.05, t$-test. Scale bar $=50 \mu \mathrm{m}$.

C30F12.4 is expressed mainly in oocytes and single-cell embryos, while its function couples oogenesis, fat homeostasis, and life span. Besides the phenotype we have found in c30f12.4 mutant worms, we also determined, using IP and MS analysis, which C30F12.4 could interact with many proteins (Fig. S5 and Table S2). Some of these proteins, such as SET-30, SRW-100, and C27B7.7, have been reported to have an important role in regulating lifespan by RNAi (Hamilton et al., 2005; Ni et al., 2012). Other proteins, like SMC-3, RPL-22, EGO-1, and so on, play some special roles in germline development (Claycomb et al., 2009; Kalis et al., 2010; Green et al., 2011), indicating that C30F12.4 may regulate oogenesis, fat metabolism, and lifespan by interacting with other proteins. However, the specific mechanism of C30F12.4 in regulating these processes and how this protein cooperates with other proteins to link these processes needs further study.

\section{MATERIALS AND METHODS}

\section{Strains}

The wild-type strain was Bristol N2. All animals were raised at $20^{\circ} \mathrm{C}$. The following alleles and transgenes were used: shg372: c30f12.4 (ust043), fog-2 (JK574), hjSi56 [vha-6p::3×FLAG-TEV-GFP::dgat-2:: let-858 3'UTR] IV, allele: ustlS039 strain: shg401 (pc30f12.4:: 3×FLAG-GFP::c30f12.4::c30f12.4 3'UTR).

\section{RNAi}

RNAi experiments were performed as described previously (Chen et al., 2014).

\section{Construction of sgRNA expression plasmids}

We manually searched for target sequences consisting of $G(N)_{19}$ NGG for c30f12.4 near the desired mutation sites. The unc-119 target sequence in the pU6::unc-119 sgRNA vector was replaced with the desired target sequence (Wiedenheft et al., 2012; Friedland et al., 2013; Chen et al., 2014). The primer sequences used for the construction of sgRNA expression plasmids are listed in Table S1.

\section{Micro-injection}

DNA mixtures were micro-injected into the gonads of young adult N2 C. elegans. For plasmids used in dual sgRNA experiments, we injected $50 \mathrm{ng} / \mathrm{mL}$ Cas 9 expressing vector, $50 \mathrm{ng} / \mathrm{mL}$ sgRNA \#1, 50 $\mathrm{ng} / \mathrm{mL}$ sgRNA \#2, and $5 \mathrm{ng} / \mathrm{mL}$ pCFJ90 vector.

\section{Screening for deletion mutants by PCR}

After injection, F1 worms which expressed mCherry were transferred to individual NGM plates. After 3 days, F1 with corresponding F2 progeny were harvested and total DNA was extracted and screened by PCR amplification with primers outside of the sgRNA-targeted regions. Mutant worms containing the deletion were singled to NGM plates and then confirmed by PCR amplification and DNA sequencing. The mutant worm strain was named shg372: c30f12.4 (ust043). The primers used for PCR screening are listed in Table S1.

3×FLAG-GFP:C30F12.4 transgene insertion by Mos1-mediated single-copy insertion (mosscl) system

MosSCI was performed as previously described (Frokjaer-Jensen et al., 2014). Briefly, the pc30f12.4::3×FLAG-GFP::c30f12.4:: c30f12.4 3'UTR construct was cloned into PCFJ151 (PCFJ151- 


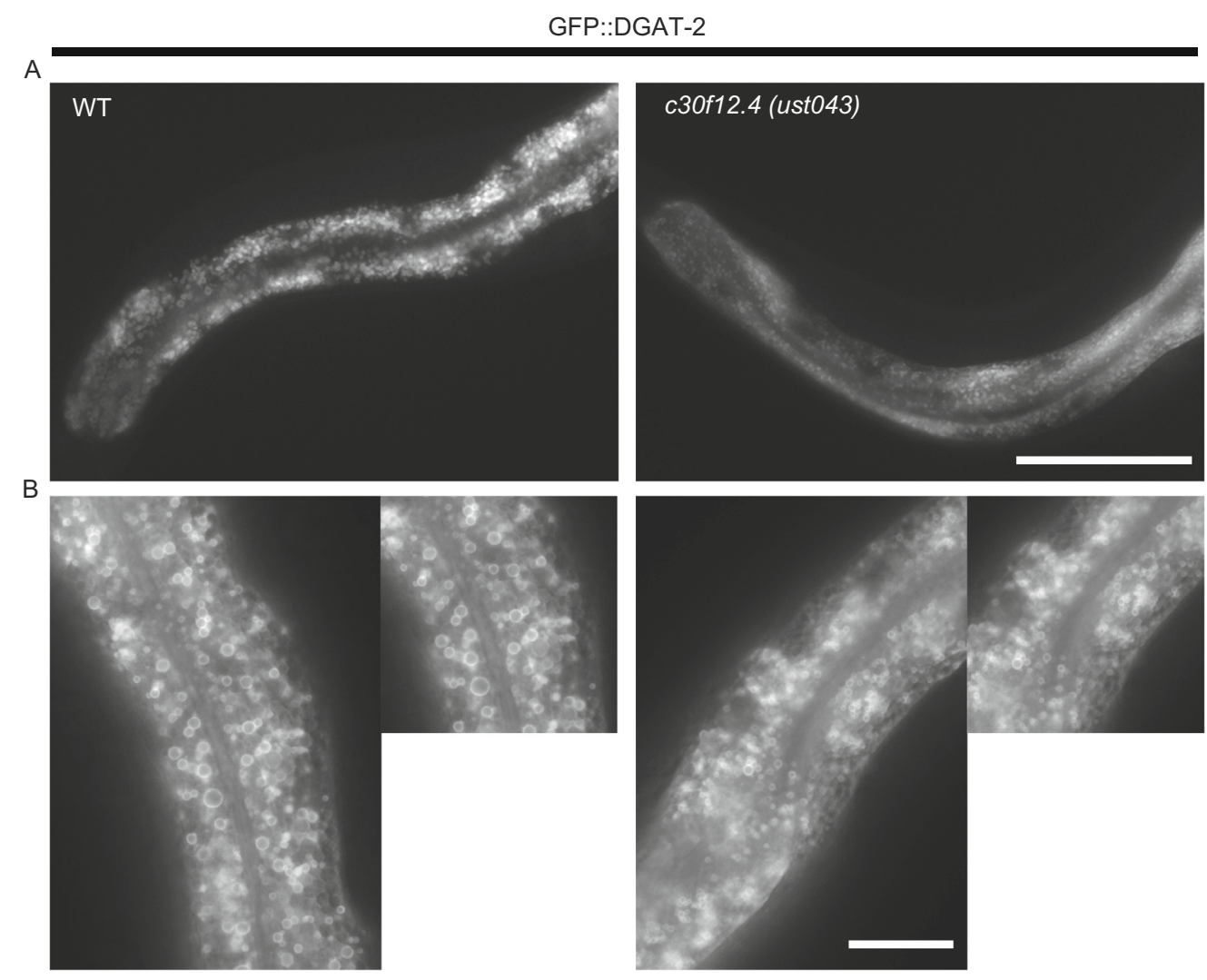

Figure 5. Loss of c30f12.4 altered fat storage in hjSi56 worms. L4 stage wild-type and c30f12.4 mutant worms on an hjSi56 background were detected directly. Images were captured using identical settings and exposure time for each image. Loss of c30f12.4 led to decreased fat storage (A) and smaller lipid droplets (B). Scale bar $=20 \mu \mathrm{m}(\mathrm{A})$ and $50 \mu \mathrm{m}(\mathrm{B})$.

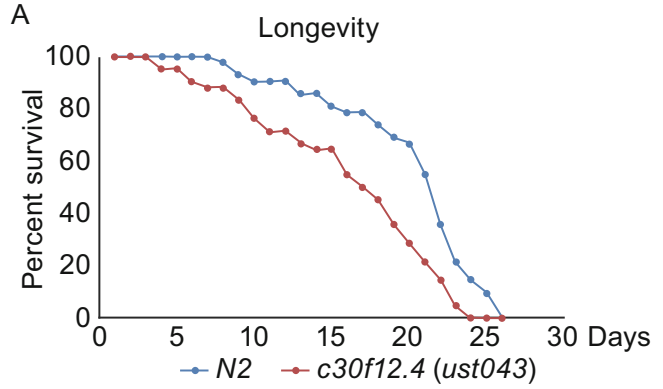

B
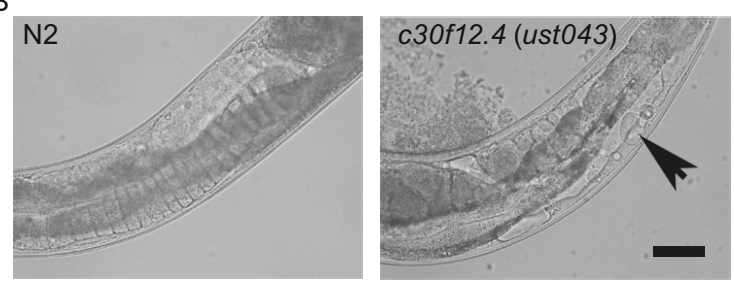

Figure 6. c30f12.4 (ust043) worms have reduced life span. (A) Adult life span of wild-type (blue line) and c30f12.4 (ust043) (red line) worms. Sixty worms per trial, average of three trials. (B) Vacuole-like structures (black arrowhead), appear in c30f12.4 (ust043) worm bodies towards the end of the reproductive period. Scale bar $=50 \mu \mathrm{m}$.
C30F12.4) and DNA mix (PCFJ151-C30F12.4 50 ng/ $\mu \mathrm{L}, \mathrm{PGH} 85 \mathrm{ng} /$ $\mu \mathrm{L}$, PCFJ90 $2.5 \mathrm{ng} / \mu \mathrm{L}$, PCFJ104 $5 \mathrm{ng} / \mu \mathrm{L}$, PJL43.1 $50 \mathrm{ng} / \mu \mathrm{L}$, Peel-1 $10 \mathrm{ng} / \mu \mathrm{L}$ ) were coinjected into the young adult strain EG4322 seeded with HT115. After injection, five worms were placed on each NGM plate and incubated at $25^{\circ} \mathrm{C}$ until starvation. Then, animals were heat-shocked for $2 \mathrm{~h}$ at $34^{\circ} \mathrm{C}$ in an air incubator, and the day after, the worm that was alive and moved well but lacked the fluorescent co-injection markers was singled. After five days, it was verified that all of the offspring moved well and identification was performed by PCR and Western blot (Fig. S6). The GFP::C30F12.4 transgene worm strain was named allele: ustlS039 strain: shg401 (pc30f12.4::3×FLAG-GFP::c30f12.4::c30f12.4 3'UTR). The primer sequences used for the construction of PCFJ151-C30F12.4 are listed in Table S1.

\section{Progeny production analysis}

Individual synchronized L4 hermaphrodites were moved to fresh plates and the number of progeny produced by each individual was counted daily until reproduction ceased for at least two days. All experiments were performed in three independent replicates at $20^{\circ} \mathrm{C}$ with at least five individuals per strain, once. 


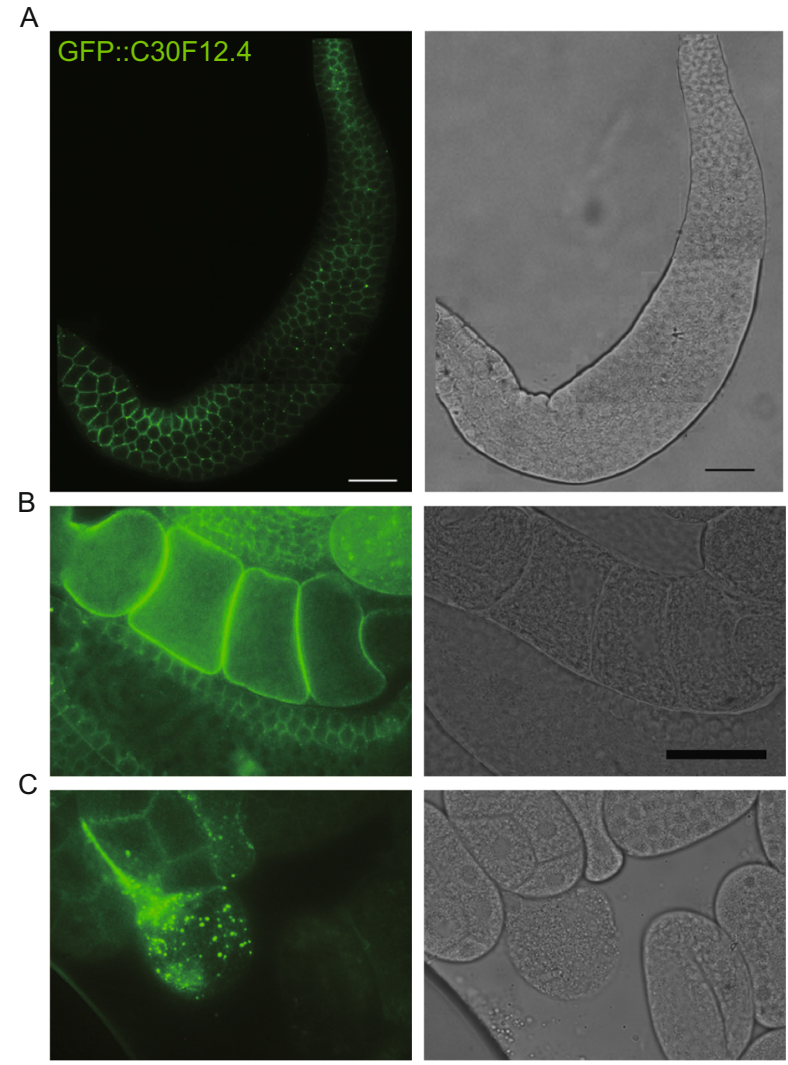

Figure 7. GFP::C30F12.4 was specifically expressed and located in germ cells and early embryos. (A and B) C30F12.4 was mainly located in the cytoplasm around the germ cells and everywhere in oocytes. (C) C30F12.4 displayed a punctate signal in single-cell embryos. Scale bar $=20 \mu \mathrm{m}$.

\section{Hatching rate}

Eggs were synchronized and allowed to develop at $20^{\circ} \mathrm{C}$ until day two of adulthood. Ten synchronized hermaphrodites were transferred to a new plate and allowed to lay eggs for $6 \mathrm{~h}$, then eggs and young adult progeny were counted. All experiments were performed in three independent replicates.

\section{Reproductive span analysis}

Individual synchronized L4 hermaphrodites were moved to fresh plates daily until reproduction ceased for at least two days. The last day of viable progeny production was noted as the day of reproduction cessation for each individual. All experiments were performed in three independent replicates at $20^{\circ} \mathrm{C}$ with at least 10 individuals per strain, once.

\section{Lifespan analysis of $C$. elegans}

Lifespan analysis was carried out at $20^{\circ} \mathrm{C}$ with worms maintained for several generations at $20^{\circ} \mathrm{C}$ on consistent dietary Escherichia coli OP50 diet ad libitum. L4 worms were transferred to fresh plates at a density of 10 worms per plate at the beginning of the experiment, day zero (Kenyon et al., 1993). No FUdR or antibiotics were included in the plates. Worms were transferred to fresh plates daily until they stopped laying eggs, after which they were transferred every three days. Animals were considered dead when they no longer responded to a gentle tap with a worm pick. The mean and maximum lifespans were determined by the average of three independent trials, each using 60 animals.

\section{Nile Red staining}

Nile Red staining was performed as previously described (Pino et al., 2013; Pang et al., 2014). Briefly, synchronized late L4 worms of the indicated genotypes were collected, washed with M9 buffer, and fixed in $40 \%$ isopropanol at room temperature for $3 \mathrm{~min}$ and stained in $3 \mathrm{mg} / \mathrm{mL}$ Nile Red (Sigma) working solution in the dark for $2 \mathrm{~h}$. Worms were then washed with M9 for $10 \mathrm{~min}$ in the dark, three times, mounted onto slides and imaged under the green fluorescent protein channel. All experiments were performed in three independent replicates with at least 20 individuals per strain, once.

Immunoprecipitation assay and mass spectrometry

Immunoprecipitation (IP) was performed as described previously (Wang et al., 2014) and mass spectrometry (MS) analysis was performed by Shanghai applied protein technology Co. Ltd.

\section{ACKNOWLEDGMENTS}

This work was supported by the National Natural Science Foundation of China (Grant No. 81430027 to F.S.), the National Basic Research Program (973 Program) (No. 2014CB943100 to F.S.), and Shanghai Municipal Education Commission-Gaofeng Clinical Medicine Grant Support (No. 20152531 to F.S.)

\section{ABBREVIATIONS}

GSC, germline stem cells; IGF-1, insulin-like growth factor; IP, immunoprecipitation; mosSCl, Mos1-mediated single-copy insertion; MS, mass spectrometry.

\section{COMPLIANCE WITH ETHICS GUIDELINES}

Lu Wang, Fei Xu, Guishuan Wang, Xiaorong Wang, Ajuan Liang, Hefeng Huang, and Fei Sun declare that they have no conflict of interest and all institutional and national guidelines for the care and use of laboratory animals were followed.

\section{AUTHOR CONTRIBUTIONS}

Lu Wang, Fei Xu, Guishuang Wang Xiaorong Wang and Ajuan Liang conducted the experiments; Fei Sun and Hefeng Huang designed the experiments; Lu Wang wrote the paper.

\section{OPEN ACCESS}

This article is distributed under the terms of the Creative Commons Attribution 4.0 International License (http://creativecommons.org/ licenses/by/4.0/), which permits unrestricted use, distribution, and 
reproduction in any medium, provided you give appropriate credit to the original author(s) and the source, provide a link to the Creative Commons license, and indicate if changes were made.

\section{REFERENCES}

Berman JR, Kenyon C (2006) Germ-cell loss extends C. elegans life span through regulation of DAF-16 by kri-1 and lipophilichormone signaling. Cell 124:1055-1068

Chen X, Xu F, Zhu C, Ji J, Zhou X, Feng X, Guang S (2014) Dual sgRNA-directed gene knockout using CRISPR/Cas9 technology in Caenorhabditis elegans. Sci Rep 4:7581

Claycomb JM, Batista PJ, Pang KM, Gu W, Vasale JJ, van Wolfswinkel JC, Chaves DA, Shirayama M, Mitani S, Ketting RF et al (2009) The Argonaute CSR-1 and its 22G-RNA cofactors are required for holocentric chromosome segregation. Cell 139:123-134

Corona G, Mannucci E, Forti G, Maggi M (2009) Hypogonadism, ED, metabolic syndrome and obesity: a pathological link supporting cardiovascular diseases. Int J Androl 32:587-598

Dernburg AF, McDonald K, Moulder G, Barstead R, Dresser M, Villeneuve AM (1998) Meiotic recombination in C. elegans initiates by a conserved mechanism and is dispensable for homologous chromosome synapsis. Cell 94:387-398

Friedland AE, Tzur YB, Esvelt KM, Colaiácovo MP, Church GM, Calarco JA (2013) Heritable genome editing in C. elegans via a CRISPR-Cas9 system. Nat Methods 10:741-743

Frokjaer-Jensen C, Davis MW, Sarov M, Taylor J, Flibotte S, LaBella M, Pozniakovsky A, Moerman DG, Jorgensen EM (2014) Random and targeted transgene insertion in Caenorhabditis elegans using a modified Mos1 transposon. Nat Methods 11:529-534

Gems D, Sutton AJ, Sundermeyer ML, Albert PS, King KV, Edgley ML, Larsen PL, Riddle DL (1998) Two pleiotropic classes of daf-2 mutation affect larval arrest, adult behavior, reproduction and longevity in Caenorhabditis elegans. Genetics 150:129-155

Goudeau J, Bellemin S, Toselli-Mollereau E, Shamalnasab M, Chen Y, Aguilaniu H (2011) Fatty acid desaturation links germ cell loss to longevity through NHR-80/HNF4 in C. elegans. PLoS Biol 9: e1000599

Green RA, Kao HL, Audhya A, Arur S, Mayers JR, Fridolfsson HN, Schulman M, Schloissnig S, Niessen S, Laband K et al (2011) A high-resolution $C$. elegans essential gene network based on phenotypic profiling of a complex tissue. Cell 145:470-482

Hamilton B, Dong Y, Shindo M, Liu W, Odell I, Ruvkun G, Lee SS (2005) A systematic RNAi screen for longevity genes in $C$. elegans. Genes Dev 19:1544-1555

Hansen M, Flatt T, Aguilaniu H (2013) Reproduction, fat metabolism, and life span: what is the connection? Cell Metab 17:10-19

Herndon LA, Schmeissner PJ, Dudaronek JM, Brown PA, Listner KM, Sakano Y, Paupard MC, Hall DH, Driscoll M (2002) Stochastic and genetic factors influence tissue-specific decline in ageing C. elegans. Nature 419:808-814

Hsin H, Kenyon C (1999) Signals from the reproductive system regulate the lifespan of $C$. elegans. Nature 399:362-366

Jia K, Albert PS, Riddle DL (2002) DAF-9, a cytochrome P450 regulating $C$. elegans larval development and adult longevity. Development 129:221-231
Judd ET, Wessels FJ, Drewry MD, Grove M, Wright K, Hahn DA, Hatle JD (2011) Ovariectomy in grasshoppers increases somatic storage, but proportional allocation of ingested nutrients to somatic tissues is unchanged. Aging Cell 10:972-979

Kalis AK, Kroetz MB, Larson KM, Zarkower D (2010) Functional genomic identification of genes required for male gonadal differentiation in Caenorhabditis elegans. Genetics 185:523-535

Kenyon CJ (2010) The genetics of ageing. Nature 464:504-512

Kenyon C, Chang J, Gensch E, Rudner A, Tabtiang R (1993) A C. elegans mutant that lives twice as long as wild type. Nature 366:461-464

Khanna A, Johnson DL, Curran SP (2014) Physiological roles for mafr-1 in reproduction and lipid homeostasis. Cell Rep 9:21802191

Klemm RW, Norton JP, Cole RA, Li CS, Park SH, Crane MM, Li L, Jin D, Boye-Doe A, Liu TY et al (2013) A conserved role for atlastin GTPases in regulating lipid droplet size. Cell Rep 3:1465-1475

Lin K, Dorman JB, Rodan A, Kenyon C (1997) daf-16: An HNF3/forkhead family member that can function to double the lifespan of Caenorhabditis elegans. Science 278:1319-1322

McCormick M, Chen K, Ramaswamy P, Kenyon C (2012) New genes that extend Caenorhabditis elegans' lifespan in response to reproductive signals. Aging Cell 11:192-202

Ni Z, Ebata A, Alipanahiramandi E, Lee SS (2012) Two SET domain containing genes link epigenetic changes and aging in Caenorhabditis elegans. Aging Cell 11:315-325

Pang S, Lynn DA, Lo JY, Paek J, Curran SP (2014) SKN-1 and Nrf2 couples proline catabolism with lipid metabolism during nutrient deprivation. Nat Commun 5:5048

Pino EC, Webster CM, Carr CE, Soukas AA (2013) Biochemical and high throughput microscopic assessment of fat mass in Caenorhabditis elegans. J Vis Exp 73(2013):e50180-e50180

Spencer WC, Zeller G, Watson JD, Henz SR, Watkins KL, McWhirter RD, Petersen S, Sreedharan VT, Widmer C, Jo J et al (2011) A spatial and temporal map of $C$. elegans gene expression. Genome Res 21:325-341

Wang MC, O'Rourke EJ, Ruvkun G (2008) Fat metabolism links germline stem cells and longevity in C. elegans. Science 322:957-960

Wang L, Liu W, Zhao W, Song G, Wang G, Wang X, Sun F (2014) Phosphorylation of CDK2 on threonine 160 influences silencing of sex chromosome during male meiosis. Biol Reprod 90:138

Wiedenheft B, Sternberg SH, Doudna JA (2012) RNA-guided genetic silencing systems in bacteria and archaea. Nature 482:331-338

Wollam J, Magner DB, Magomedova L, Rass E, Shen Y, Rottiers V, Habermann B, Cummins CL, Antebi A (2012) A novel 3-hydroxysteroid dehydrogenase that regulates reproductive development and longevity. PLoS Biol 10:e1001305

Xu N, Zhang SO, Cole RA, McKinney SA, Guo F, Haas JT, Bobba S, Farese RV Jr, Mak HY (2012) The FATP1-DGAT2 complex facilitates lipid droplet expansion at the ER-lipid droplet interface. J Cell Biol 198:895-911 